\title{
The impact of genotyping error on family-based analysis of quantitative traits
}

\author{
Gonçalo R Abecasis ${ }^{1}$, Stacey S Cherny ${ }^{1}$ and Lon R Cardon*,1 \\ ${ }^{1}$ Wellcome Trust Centre for Human Genetics, University of Oxford, Oxford, UK
}

Errors in genotyping can substantially influence the power to detect linkage using affected sib-pairs, but it is not clear what effect such errors have on quantitative trait analyses. Here we use Monte Carlo simulation to examine the influence of genotyping error on multipoint vs two-point analysis, variable map density, locus effect size and allele frequency in quantitative trait linkage and association studies of sib-pairs. The analyses are conducted using variance components methods. We contrast the effects of error on quantitative trait analyses with those on the affected sib-pair design. The results indicate that genotyping error influences linkage studies of affected sib pairs more severely than studies of quantitative traits in unselected sibs. In situations of modest effect size, $5 \%$ genotyping error eliminates all supporting evidence for linkage to a true susceptibility locus in affected pairs, but may only result in a loss of $15 \%$ of linkage information in random pairs. Multipoint analysis does not suffer substantially more than two-point analysis; for moderate error rates $(<5 \%)$, multipoint analysis with error is more powerful than two-point with no error. Map density does not appear to be an important factor for linkage analysis. QTL association analyses of common alleles are reasonably robust to genotyping error but power can be affected dramatically with rare alleles. European Journal of Human Genetics (2001) 9, 130-134.

Keywords: linkage analysis; genotype error; association analysis; SNP; QTL

\section{Introduction}

In studies of monogenic traits and in the construction of genetic maps, errors in genotyping can mislead inferences of map order and distance ${ }^{1-4}$ and incorrect estimates of allele frequency can mislead statistical inference. ${ }^{5}$ For complex trait studies, the effects of genotype error are less well characterised. By definition, multifactorial traits result from the effects of multiple genetic loci of relatively small effect, each of unknown gene action and potentially involved in uncharacterised interactions with other loci and environmental factors. Thus, it is not clear what impact genotyping error might have on the detection of any particular locus.

Recently, Douglas et $a l^{6}$ showed that even a slight amount of genotyping error could induce a substantial loss of

*Correspondence: Lon $\mathrm{R}$ Cardon, PhD, Wellcome Trust Centre for Human Genetics, University of Oxford ,Roosevelt Drive, Oxford OX3 7BN, United Kingdom. Tel: +44 1865287 503; Fax: +44 1865287 650; E-mail: lon.cardon@well.ox.ac.uk

Received 20 July 2000; revised 20 September 2000; accepted 29 September 2000 information in studies of affected sib-pairs (ASP). Given that detection of complex trait loci may require thousands of sibpairs, ${ }^{7,8}$ most ongoing studies have, at best, marginal power for mapping trait loci. Thus, it is important that $0.5-1 \%$ genotype error, which would be deemed exemplary in many laboratories, ${ }^{9,10}$ has the potential to mask medically important findings from otherwise sufficiently powered genetic studies.

Many complex traits of current interest are quantitative in scale, including obesity, osteoporosis/bone density, asthma/ allergy, intermediate phenotypes of coronary heart disease and NIDDM, and dyslexia. It is not clear that studies of quantitative traits would follow the same patterns of power loss as ASPs, as they make use of IBD information in different ways. It would be useful to know the extent to which quantitative trait studies are influenced by typing error, particularly in the context of the emerging SNP map, ${ }^{11}$ which will permit genotyping of thousands of markers on familybased samples. Here we conduct a series of simulation studies to evaluate the impact of genotyping error on studies of quantitative traits in unselected sib-pairs. We compare the 
influence of error on quantitative trait locus (QTL) studies with those of affected sib-pairs with respect to multipoint $v s$ two-point analysis, marker density, locus effect size and allele frequency. We also examine the impact of genotyping error on the power of family-based association studies, following the recent interest in association models for quantitative traits (see Abecasis et $a l^{12}$ ).

\section{Methods}

For all simulations, results were averaged over 1000 replicates of 1000 two-sib families with no parents. The quantitative trait simulations and data analyses were conducted using a variance components approach for continuous phenotypes in unselected sibling pairs as recently described. ${ }^{13}$ For ASP analyses, sib-pairs were simulated under several recurrencerisk ratios, $\lambda_{\mathrm{s}}{ }^{14}$ with lod scores calculated using the allelesharing methods developed by Kong and Cox, ${ }^{15}$ incorporating the sign of the effect. For multipoint analysis, we considered either a sparse map of 10 4-allele markers separated by $10 \mathrm{cM}$, or a dense map of 50 SNP markers separated by $2 \mathrm{cM}$ intervals. In all cases we simulated a trait locus half-way between the middle two markers. The Haldane mapping function was used to relate map distance and recombination fraction. Identity-by-descent (IBD) estimates were calculated using the Lander-Green algorithm, ${ }^{16}$ incorporating all available marker data and the simulated map order and distances. For two-point analyses, a single 4-allele marker located close to the trait locus $(\theta=0.05)$ was simulated, with IBD information derived from the marker only. All marker alleles were of equal frequency and were in initial Hardy-Weinberg and linkage equilibrium (except for the association assessments).

Genotype errors were induced by randomly re-sampling two alleles from a distribution of equally frequent alleles (which included the original, correct, genotype), according to a prescribed random error rate of $0.5 \%, 1 \%, 2 \%, 5 \%$ and $10 \%$. This error induction strategy is analogous to the 'genotype error model' of Douglas et al. ${ }^{6}$ The linkage tests were conducted using the QTDT program, ${ }^{13}$ with lods calculated as likelihood-ratio $\chi^{2} /[2 \ln (10)]$. A purpose-written program implementing the Kong and Cox linkage test ${ }^{15}$ was used to calculate lod scores for the ASP analyses.

The variance components approach used for the linkage analyses is also appropriate for association studies. ${ }^{13,17}$ For comparison with the linkage results, a series of association simulations and analyses were conducted. Complete linkage disequilibrium between a QTL and a neighbouring marker locus $\left(\mathrm{D}^{\prime}=1,(\theta=0)\right.$ was introduced in the parental chromosomes (which were later omitted from analysis) and randomly segregated to the siblings. The QTL was simulated to explain $2 \%$ of the phenotypic variance and had equally frequent alleles. The residual sibling correlation was 0.50 . Allele frequencies of the marker locus were varied to explore the interaction of allele frequency and genotyping error. All association analyses were conducted using the model of Fulker et al, ${ }^{17}$ which provides a quantitative-TDT analogue that makes use of siblings with no parents.

\section{Results}

A comparison of the effects of genotype error on multipoint and two-point linkage analysis is shown in Table 1. For randomly ascertained siblings with continuous phenotypes, the results indicate that the proportional reduction in average lod score is the same whether the data are analysed by two-point or multipoint methods. Indeed, multipoint analysis in the presence of $10 \%$ genotyping error is still more powerful than two-point analysis with no error at all (multipoint average lod $=1.53$; two-point lod $=1.40$ ). These results point to the general utility of multipoint analysis of unselected sibling pairs, even in the presence of genotyping error. While multipoint analysis can clearly facilitate detection of genotyping error, ${ }^{6,10}$ it does not suffer a greater loss of power to detect genetic influences on continuous characters than two-point analysis.

For affected sib-pairs, genotype error has a greater impact on multipoint outcomes than on two-point results. In this case, multipoint analysis in the presence of $1 \%$ typing error would lose $6 \%$ more information than would a two-point

Table 1 Proportion of average lod retention in the presence of genotype error: multipoint $v s$ two-point analysis ${ }^{a}$

\begin{tabular}{|c|c|c|c|c|c|}
\hline \multirow[b]{2}{*}{ Error rate } & \multicolumn{2}{|c|}{ Quantitative trait/random sib-pairs } & \multicolumn{3}{|c|}{ Discrete trait/affected sib-pairs } \\
\hline & Multipoint & Two-point & Multi & ooint & Two-point \\
\hline $0 \%$ & $1.00(2.28)$ & $1.00(1.40)$ & 1.00 & $(19.20)$ & $1.00(12.70)$ \\
\hline $2 \%$ & $0.93(2.12)$ & $0.93(1.30)$ & 0.56 & $(10.75)$ & $0.66(8.38)$ \\
\hline $5 \%$ & $0.84(1.92)$ & $0.78(1.09)$ & 0.15 & $(2.88)$ & 0.24 \\
\hline $10 \%$ & $0.67(1.53)$ & $0.67(0.94)$ & 0.00 & $(-0.85)$ & $0.02 \quad(0.25)$ \\
\hline
\end{tabular}

aThe 'average lod retained' refers to the ratio of the average lod score obtained under each specific level of genotype error to the average lod score obtained in the absence of error. Average lods (including the sign of the effect) are shown in parentheses. The multipoint data comprised 10 markers spaced at $10 \mathrm{~cm}$ intervals, with the trait locus in the centre. For the quantitative trait, the biometrical model involved a di-allelic, equifrequent, trait locus accounting for $20 \%$ of the phenotypic variance, with a residual sibling correlation of 0.05 . For the affected sib-pairs, a $\lambda_{s}$ of 2.0 was modelled. 
evaluation. At $2 \%$ error or greater, this differential is greater, as multipoint analysis of ASP data would reduce the average lod by approximately $10 \%$ more than would two-point analysis of the same data. Nevertheless, despite the greater loss in information, for error rates $<5 \%$, multipoint is still more powerful than two-point analysis.

ASPs and random sib-pairs also differ substantially with respect to the extent of impact from genotyping error. The results in Table 1 indicate that for a quantitative trait in unselected pairs, the effect of genotype error is not extensive until the error rate reaches high levels. Error rates up to $2 \%$ reduce the average lod no more than $9 \%$, while an excessive error rate of $10 \%$ reduces the average lod by approximately $33 \%$.

The effects of typing error on ASPs are generally more severe than those for unselected sibs. In the results of Table 1 (locus $\lambda_{\mathrm{s}}=2.0$ ), $2 \%$ genotype error reduces the expected lod score by nearly $50 \%$ and higher levels of error virtually eliminate the linkage signal: at 5\% error, nearly $90 \%$ of the average lod score is lost, and at $10 \%$ error the expected lod is zero. Unlike the results for quantitative traits, which are essentially independent of the additive genetic variance of the locus (data not shown), these results are strongly dependent on the effect size of the locus (described below).

The simulation results in Figure 1 show the effects of genotype error on detection of a QTL using a sparse $(10 \mathrm{cM}$; top panel) or dense ( $2 \mathrm{cM}$; bottom panel) marker map, in which the curves reflect the decrease in lod according to successive increases in genotyping error. These results again show the relatively minor effect of genotyping error rates up to $2 \%(<10 \%$ loss in expected lod). The clear similarity between the two panels in Figure 1 also shows that the effects of genotype error are basically independent of map density, an outcome previously observed in affected sib-pairs. ${ }^{6}$ In both map configurations, increasing error naturally results in less precision of QTL location, as the $95 \%$ confidence interval for the location of the linkage peak expands by $7 \%, 24 \%$ and $40 \%$ for error rates of $1 \%, 5 \%$ and $10 \%$, respectively.

For comparison of the QTL results shown in Figure 1, a series of simulations of ASP were conducted. The results of these simulations, presented in Figure 2, indicate that genotype error can have a much greater impact on ASP studies than on those involving unselected sibs. When the effect of the locus is relatively small (eg, $\lambda_{s}=1.25$; Figure 2, top panel), a study of 1000 ASPs yielded an average peak lod score of 2.90 in the absence of genotyping error. Introduction of $1 \%$ error reduced this lod score to 1.40 ; ie, less than $50 \%$ of its original value. Interestingly, higher error rates actually yield negative average lods using the Kong and $\operatorname{Cox}^{15}$ approach; for example a $10 \%$ error rate yields all average lod scores $<-8.5$. Loci of larger effect, shown in the middle $\left(\lambda_{s}=2.0\right)$ and bottom panels $\left(\lambda_{s}=4.0\right)$ of Figure 2 , reveal the same general trend, but are less severely influenced by a typing error. For example, the results in the bottom panel show that $1 \%$ genotyping error on a region yielding a sib
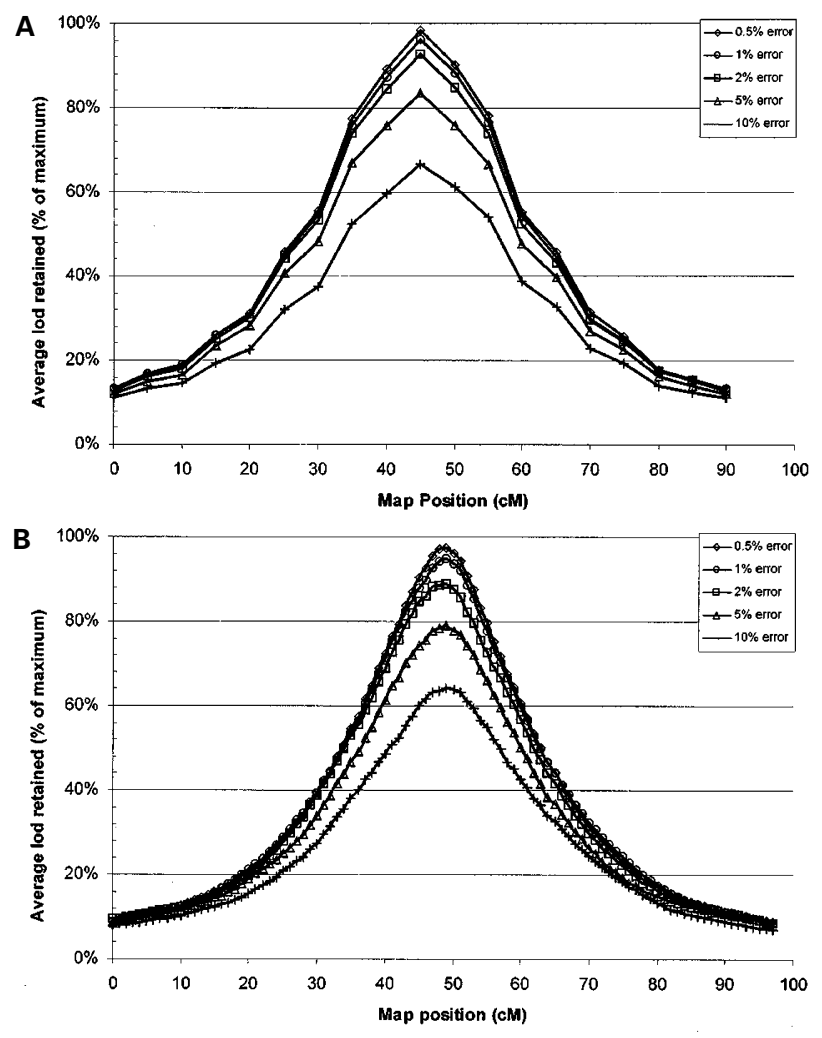

Figure 1 Comparison of the effects of genotyping error on QTL detection using (A) sparse and (B) dense marker maps. The sparse map in panel $A$ was constructed using $10 \times 4$-allele markers spaced $10 \mathrm{~cm}$ apart. The dense map in panel $\mathbf{B}$ comprised 50 diallelic markers at $2 \mathrm{~cm}$ intervals. All markers had equally frequent alleles. The successive curves, from top to bottom within each panel, describe the proportion of the peak average lod for genotype error rates of $0.5 \%, 1 \%, 2 \%, 5 \%$ and $10 \%$, respectively.

recurrence risk ratio of 4.0 only reduces the average lod by $15 \%$. Even in this case, however, $10 \%$ error virtually eliminates any evidence for linkage.

The effects of genotyping error on association analyses of unselected families are summarised in Figure 3. For common alleles (>20\% frequency) and modest genotype error $(\leqslant 2 \%)$ the loss of statistical power is not excessive. In all such cases at least $90 \%$ of the original lod score is retained. However, for less frequent alleles, genotyping error can have severe consequences. Analysis of an allele having 5\% frequency but genotyped with only $1 \%$ error reduces the average lod of the study by more than $20 \%$. A $5 \%$ error rate would cost such a study $55 \%$, while $10 \%$ would result in a loss of more than $70 \%$ of the original lod score. Thus, while genotyping error does not seem of great concern for common associated alleles, it is paramount for rare alleles. These outcomes do not appear attributable to specific properties of TDT tests (eg, reliance on heterozygous parents), as analysis of a population-based test of mean differences by genotype yielded 

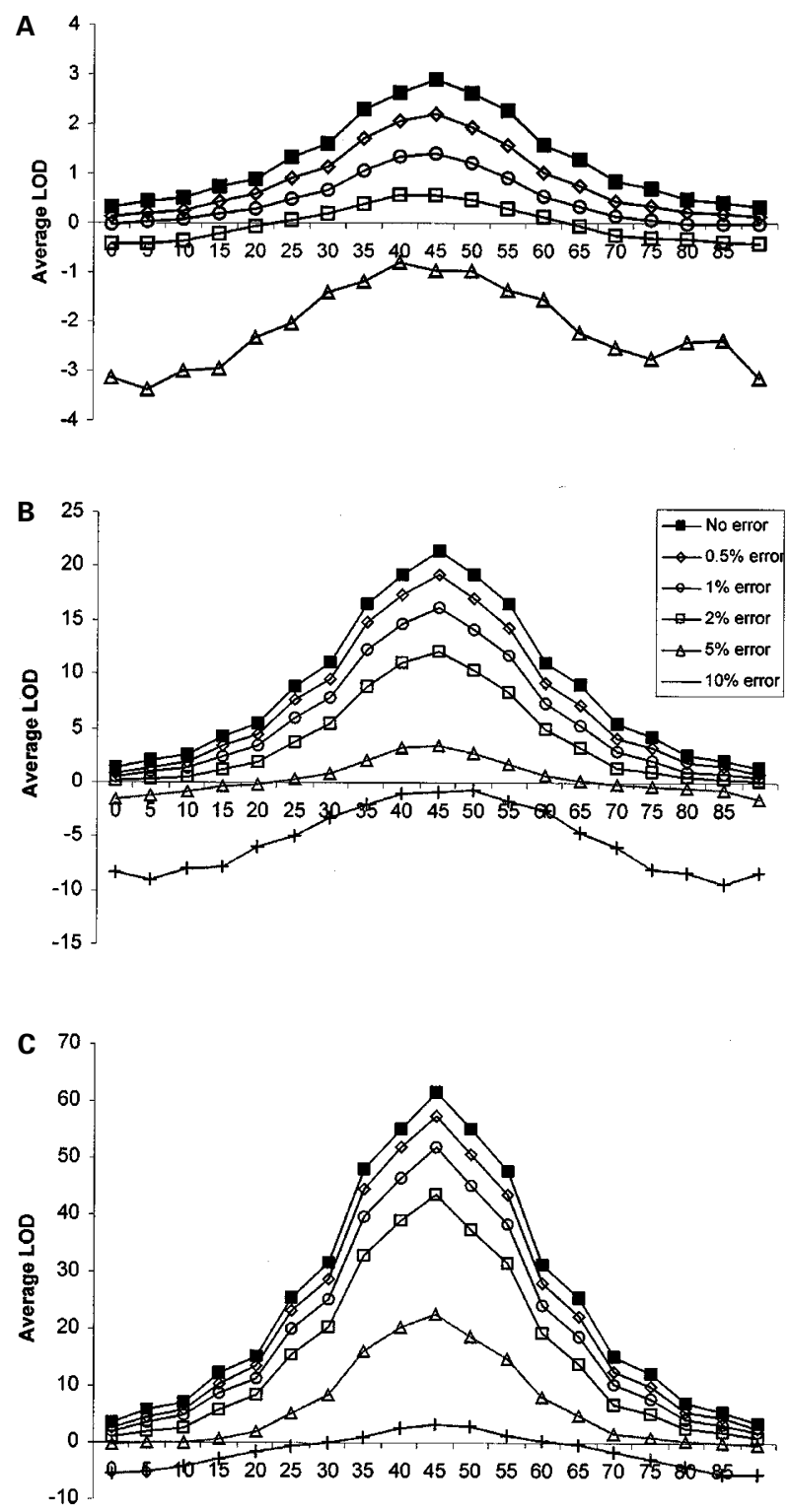

Figure 2 Comparison of the effects of genotyping error on disease-locus detection under different locus effect sizes. The results in $(\mathbf{A}-\mathbf{C})$ were obtained from affected sib-pair simulations of loci having $\lambda_{s}=1.25,2.0$ and 4.0, respectively. The (descending) successive curves represent the average lod scores according to genotype error rates of $0 \%, 0.5 \%, 1 \%$, $2 \%, 5 \%$ and $10 \%$, respectively. The markers were simulated under the $10 \mathrm{~cm}$ spacing described in the text. Results from dense map simulations were not substantially different from those presented (data not shown). In panel A, the $10 \%$ error rate data are not shown; all data points in this case had average lod scores $<-8.5$.

indistinguishable results as those presented in Figure 3 (data not shown). Thus, in the case of genome-screens for association, the effects of genotype error could easily

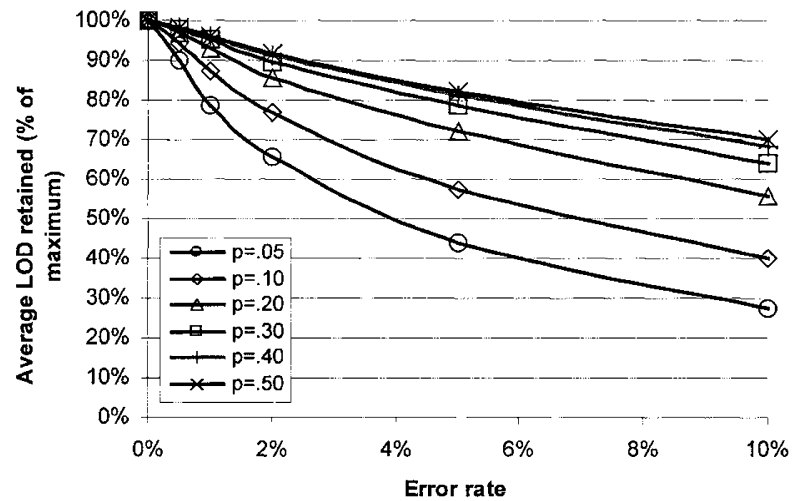

Figure 3 Effects of genotyping error on QTL association. The successive curves represent the results for marker and QTL allele frequencies of $5 \%, 10 \%, 20 \%, 30 \%, 40 \%$ and $50 \%$.

dominate the calculations of required sample sizes during study design.

\section{Discussion}

In general, these simulation results for linkage and association studies suggest that even low rates of genotyping error can substantially impact the power of a study and thereby alter the outcomes and conclusions. For quantitative characters in random sib-pair samples, genotyping error impacts multipoint and two-point methods of analysis approximately equally and independently of effect size. In contrast, the loss of power in ASP studies is greater in multipoint analyses and for loci of small effect size. Unfortunately, such loci may be more characteristic than exceptional for multifactorial traits, thus emphasising the importance of accurate genotyping and efficient error detection.

Our comparison of ASPs and randomly ascertained sibpairs is intentionally extreme, owing to prior expectations of differential loss of power in the two study designs. Of primary importance is the difference in treatment of allele-sharing information in the two strategies. In particular, ASP designs rely on mean allele sharing, while QTL analyses in unselected sib-pairs are based on sibling correlations (or covariances) between phenotypic similarity and allele sharing. It might be expected that tests that incorporate the full allele-sharing distribution would not be affected in the same way as those which rely exclusively on average sharing.

This difference in the usage of allele-sharing information is relevant in comparing the impact of error on multipoint and two-point analysis. Multipoint analyses combine information from different markers and thus might be expected to compound the effects of genotyping error, since error at one marker can effectively reduce power at a linked marker that is itself error-free. However, this may affect mean allele sharing and the total allele sharing distribution differently. For example, using the quantitative data summarised in Table 
1 , induction of $5 \%$ genotyping error reduces the average sharing from $50 \%$ to $45 \%$ in the multipoint case, but only from $50 \%$ to $48 \%$ in the two-point case. In contrast, the correlation between allele sharing in the presence and absence of error is identical in both multipoint and twopoint models $(\mathrm{r}=0.88)$. Thus, QTL analyses in unselected sibpairs would suffer similarly to ASPs if only average allele sharing were used in the test, but appear unaffected when analysed using the covariance modelling approach.

The use of mean allele sharing in ASPs also contributes to the relationship between genotype error and locus effect size (Figure 2). Tests that use mean allele sharing to infer linkage do not maintain a linear relationship between average sharing and the lod statistic. Consequently, the lod-score effects from any perturbation of the mean, such as that induced by genotyping error, will depend on the initial (true) mean value. The present results indicate that this does not hold in the case of random sib-pairs. Thus, in general, it appears that much of the ASP vs QTL differences can be ascribed to the differential usage of IBD data in the test statistic.

The strong influence of IBD information implies that different selection strategies for quantitative traits would not be identically influenced by genotyping error. For example, the discordant sib-pair design advocated by Risch and Zhang $^{18}$ would likely suffer inflation of Type I error because, as noted previously, most typing errors lead to decreases in IBD, yet decreased sharing forms the basis for (positive) linkage evidence in the discordant sib design. Conversely, concordant pairs might suffer more a loss of power, owing to reliance on increased allele sharing akin to the ASP situation. Interestingly, one of the most commonly-used strategies, that of single selection (extreme proband and unselected siblings), effectively includes both discordant and concordant pairs in the selected tail of the distribution. The influence of genotyping error on this design would most likely depend on the type of analysis performed, particularly in regard to the usage of average allele sharing vs sibling covariance.

It should be noted that we have selected an extreme study design of siblings with no parental information, thereby precluding detection of any Mendelian inconsistencies. We have also made use of the known (simulated) map order and distances in an attempt to minimise additional effects of map error that would accompany map estimation from the data at hand. Further studies are needed to determine the influence of genotyping error on different family designs and genetic map information.

In general, the ASPs and randomly ascertained sib-pairs evaluated here illustrate a broad range of effects: in some cases (eg, QTL studies of linkage and of association with common alleles), modest error rates may be tolerable for a specific study design; in other cases, however (eg, ASP studies of loci of small effect size, QTL association studies of uncommon alleles), modest error rates can have devastating effects on power. With the current increase in SNP marker availability and likelihood of extensive genotyping throughput, family studies would benefit from careful consideration of genotyping error in developing robust study designs and calculating power.

\section{Acknowledgements}

The authors are supported by the Wellcome Trust; GRA is a Wellcome Prize student. This work was supported in part by grant EY-12562 from the National Eye Institute, National Institutes of Health, USA. We wish to thank two anonymous reviewers for their helpful comments.

\section{References}

1 Buetow KH: Influence of aberrant observations on highresolution linkage analysis outcomes. Am J Hum Genet 1991; 49: $985-994$.

2 Ott J: Analysis of Human Genetic Linkage, (Johns Hopkins University Press, Baltimore, 1991).

3 Lincoln SE, Lander ES: Systematic detection of errors in genetic linkage data. Genomics 1992; 14: 604-610.

4 Shields DC, Collins A, Buetow KH, Morton NE: Error filtration, interference, and the human linkage map. Proc Natl Acad Sci USA 1991; 88: 6501-6505.

5 Ott J: Strategies for characterizing highly polymorphic markers in human gene mapping. Am J Hum Genet 1992; 51: 283-290.

6 Douglas JA, Boehnke M, Lange K: A multipoint method for detecting genotyping errors and mutations in sibling-pair linkage data. Am J Hum Genet 2000; 66: 1287-1297.

7 Risch N, Merikangas K: The future of genetic studies of complex human diseases. Science 1996; 273: 1516-1517.

8 Risch NJ: Searching for genetic determinants in the new millennium. Nature 2000; 405: 847 -856.

9 Brzustowicz LM, Merette C, Xie X et al. Molecular and statistical approaches to the detection and correction of errors in genotype databases. Am J Hum Genet 1993; 53: 1137-1145.

10 Goring HH, Terwilliger JD: Linkage analysis in the presence of errors II: marker-locus genotyping errors modeled with hypercomplex recombination fractions. Am J Hum Genet 2000; 66: $1107-1118$.

11 Roberts L: Human genome research. SNP mappers confront reality and find it daunting. Science 2000; 287: 1898 - 1899.

12 Abecasis GR, Cookson WO, Cardon LR: Pedigree tests of transmission disequilibrium. Eur J Hum Genet 2000; 8: 545 - 551.

13 Abecasis GR, Cardon LR, Cookson WO: A general test of association for quantitative traits in nuclear families. $A m J$ Hum Genet 2000; 66: 279-292.

14 Risch N: Linkage strategies for genetically complex traits. I. Multilocus models. Am J Hum Genet 1990; 46: 222-228.

15 Kong A, Cox NJ: Allele-sharing models: LOD scores and accurate linkage tests. Am J Hum Genet 1997; 61: 1179-1188.

16 Lander ES, Green P: Construction of multilocus genetic linkage maps in humans. Proc Natl Acad Sci, USA 1984; 84: 2363-2367.

17 Fulker DW, Cherny SS, Sham PC, Hewitt JK: Combined linkage and association sib-pair analysis for quantitative traits. Am J Hum Genet 1999; 64: 259-267.

18 Risch N, Zhang H: Extreme discordant sib pairs for mapping quantitative trait loci in humans. Science 1995; 268: 15841589. 